\title{
Topological horseshoe in Chen system with time delay
}

\author{
Hai-Peng Ren · Kun Tian · Celso Grebogi
}

Received: date / Accepted: date

\begin{abstract}
A single-scroll attractor is studied through both simulations and experiments in the Chen system with time delay. The proof that the attractor is chaotic is not a trivial task, especially for a time delay system. One approach to prove the occurrence of chaos is to use a topological horseshoe and its corollary if the geometrical relationship of the attractor on a Poincaré section satisfies certain conditions. This paper presents two methods for finding the horseshoe in a Chen system with time delay. In the first method, we construct a Poincaré section, select two quadrilaterals, and calculate the location relationship of the quadrilaterals under the Poincaré map. In the second method, we select quadrilaterals in the neighborhood of a short unstable periodic orbit on the Poincaré section and obtain the approximate location of the quadrilaterals under the map and the reverse map. By using the above two methods, the geometrical expansion of the quadrilaterals under the map satisfies the topological horseshoe corollary. Thus, we prove that the single-scroll attractor is indeed chaotic.
\end{abstract}

Keywords Chen system with time delay $\cdot$ single-scroll hyper-chaotic attractor · Poincaré map · topological horseshoe

\section{Introduction}

One of the challenges in the field of nonlinear dynamics research is the dynamic analysis of its complex behavior. The Smale's profound paper was triggered by the paper of seminal earlier works of Levinson, in which he written that the strange attractor contains infinitely many periodic orbits [1]. In 1967, Smale proposed the Smale horseshoe [2]. The proof of its existence in the dynamics is the proof of the presence of chaos. Since then, the Smale horseshoe theory has been developed further and used in combination with symbolic dynamics prove the existence of chaos rigorously. However, the Smale horseshoe theory remains difficult to be applied because of the strict conditions and the large amount of rigorous numerics required [3]. The properties of Lorenz attractor was studied by Colin Sparrow [4] and M.Rychlik [5]. In 1999, Tucker was the first to strictly prove that the Smale horseshoe exists in a Lorenz system [6]. Kennedy and Yorke developed the topological horseshoe theory [7] based on the Smale horseshoe; however, it is difficult to satisfy the preconditions of their theorem.

Hai-Peng Ren · Kun Tian

Shaanxi Key Lab. of Complex System Control and Intelligent Information Processing, Xi'an University of Technology, Xian 710048, China

Tel.: $+123-45-678910$

Fax: +123-45-678910

E-mail: renhaipeng@xaut.edu.cn(Hai-Peng Ren)

Celso Grebogi

Xi'an University of Technology, Xian 710048, China

Institute for Complex System and Mathematical Biology, Aberdeen, AB24 3UE, United Kingdom 
In 2004, based on the Smale horseshoe theory, Yang and Tang derived the Topological Horseshoe Lemma [8]; however, their method requires many numerical trials and considerable computational cost. To find the topological horseshoe more easily, a topological horseshoe corollary was proposed in [9]. By using the topological horseshoe corollary, the topological horseshoe in a shunted nonlinear resistive-capacitive-inductance junction (RCLSJ) model [10] and several types of 3D chaotic systems [11-18], fractional order system [19], and 4D hyperchaotic system [20] were investigated. For a hyper-chaotic Rössler system, a two-dimensional topological expanding in four-dimensional space was studied in [21] to obtain the topological horseshoe. For a threedimensional hyper-chaotic system, the dimension was reduced along the contracting direction into a 2D plane projection [22]; the two-dimensional topological horseshoe was shown in the new 2D system, thus showing that the topological horseshoe existed in the original 3D dimension. The system with time delay is theoretically infinite dimensional, which might be more relevant in science and in engineering applications [23-26]. There are few results about topological horseshoes in nonlinear systems with time delay. In this paper, we prove the existence of a topological horseshoe in the Chen system with time delay. The distinctive characteristics of the analysis is to show the topological mechanism producing the chaotic attractor in the Chen system with time delay. The chaotic characteristics of infinite dimensional systems is proved by utilizing the topological horseshoe theorem, and thus may attract interest to other infinite dimensional systems and thus promoting their future technological applications.

In this paper, the topological horseshoe Corollary is applied in a time delay nonlinear system. We use the Poincare mapping to reduce the dimension; we then obtain the contracting and expanding mapping of the quadrilaterals, which satisfy the conditions of the Topological Horseshoe Lemma, to identify the topological horseshoe. Finally, we show that the single-scroll attractor is indeed a chaotic attractor, as made evident through both simulation and experimental results in the Chen system with time delay.

The remainder of this paper is organized as follows. In Section 2, simulations and physical experiments of a single-scroll attractor in a Chen system with time delay are given. In Section 3, the Topological Horseshoe Lemma and its Corollary are revisited. In Section 4, the topological horseshoe is identified for the chaotic attractor in the Chen system with time delay. Two numerical methods are used to find the topological horseshoe. Finally, the conclusions are presented in Section 5.

\section{Chen system with time delay}

The Chen system is given as follows [27]:

$$
\left\{\begin{array}{l}
\dot{x}(t)=a(y(t)-x(t)) \\
\dot{y}(t)=(c-a) x(t)-x(t) z(t)+c y(t) . \\
\dot{z}(t)=x(t) y(t)-b z(t)
\end{array}\right.
$$

For the parameters $a=35, b=3$, and $c=18.35978$, three equilibria of the system are $O_{0}=(0,0,0)$, $O_{+}=\left(x_{0}, y_{0}, z_{0}\right)$, and $O_{-}=\left(-x_{0},-y_{0}, z_{0}\right)$, where $x_{0}=y_{0}=\sqrt{b(2 c-a)}=2.2713 ; z_{0}=2 c-a=1.7196$. The Jacobi matrix of the Chen system for the equilibrium $O_{+}$is

$$
J=\left[\begin{array}{ccc}
-a & a & 0 \\
c-a-z & c & -x \\
y & x & -b
\end{array}\right] .
$$

The characteristic polynomial is

$$
\lambda^{3}+(a+b-c) \lambda^{2}+b c \lambda+4 a b c-2 a^{2} b=0 .
$$

The eigenvalues of equation (3) are $\gamma_{1}=-17.6801, \sigma_{1,2} \pm \omega_{1,2} i=-0.9801 \pm 4.4118 i$. Therefore, the equilibria $\mathrm{O}_{+}$and $\mathrm{O}_{-}$are stable.

The Jacobi matrix of the Chen system for the equilibrium $O_{0}$ is 


$$
J=\left[\begin{array}{ccc}
-a & a & 0 \\
c-a & c & 0 \\
0 & 0 & -b
\end{array}\right]
$$

The characteristic polynomial is

$$
\lambda^{3}+(a+b-c) \lambda^{2}+\left(a^{2}+a b-b c-2 a c\right) \lambda-2 a b c+a^{2} b=0 .
$$

The eigenvalues of equation (5) are $\lambda_{1}=-3, \lambda_{2}=-19.6959, \lambda_{3}=3.0557$. Therefore, $O_{0}$ is an unstable equilibrium of system (1).

From the above analysis, we conclude that the Chen system is stable for $a=35, b=3$, and $c=18.35978$. By introducing direct time delay feedback control into the equations, we obtain Chen system with time delay $[28,29]$ :

$$
\left\{\begin{array}{l}
\dot{x}(t)=a(y(t)-x(t)) \\
\dot{y}(t)=(c-a) x(t)-x(t) z(t)+c y(t) \\
\dot{z}(t)=x(t) y(t)-b z(t)+k(z(t)-z(t-\tau))
\end{array},\right.
$$

where $k$ is the delay feedback gain and $\tau$ is the delay time.

References [28] and [29] showed that double-scroll attractors can be produced in (6). On the other hand, in this paper, we show that the system can produce a single-scroll attractor with time delay parameters $k=2.85$ and $\tau=0.3$, as shown in Fig. 1. The corresponding experimental results of the single-scroll attractor are shown in Fig. 2.

By calculating the Lyapunov exponents, we identify that the Chen system with $a=35, b=3, c=18.35978$, $k=2.85$, and $\tau=0.3$ has three positive Lyapunov exponents equal to $1.0089,0.3046$ and 0.0048 . Therefore, the single-scroll attractor is a hyper-chaotic attractor. To prove that the attractor is indeed chaotic, we obtain the topological horseshoe to show the existence of chaos in the system.

\section{Preliminaries of topological horseshoe theory}

\subsection{A Brief Theory of Symbolic Dynamics}

We first revisit some basic concepts of symbolic dynamics [30].

Let $A=\left\{a_{1}, a_{2}, \cdots, a_{N}\right\}$ be a collection of finite elements, where $A=\left\{a_{1}, a_{2}, \cdots, a_{N}\right\}$ is the alphabet. The letters $a_{j}(j=1,2, \cdots, N)$ are called symbols. The distance matrix on $A$ is defined as

$$
d\left(a_{i}, a_{j}\right)= \begin{cases}0 & \text { if }, i=j \\ 1 & \text { if }, i \neq j\end{cases}
$$

Let $\sum_{A}$ be the collection of all bi-infinite sequences given as $S=\left(\cdots, s_{-2}, s_{-1} ; s_{0}, s_{1}, s_{2}, \cdots\right)$, where $s_{j} \in A($ $j=0, \pm 1, \pm 2, \cdots)$. The sign ";", is added at the left-hand side of the element with zero sub-index. The distance between two sequences $S, T$ is defined on $\sum_{A}: \forall S, T \in \Sigma_{A}$.

$$
d(S, T)=\sum_{j=-\infty}^{\infty} \frac{d\left(s_{j}, t_{j}\right)}{2^{|j|}}
$$

With respect to metric (7), $\sum_{A}$ is a compact, totally disconnected metric space. Next, an $m$-shift map $\sigma: \sum_{A} \rightarrow \sum_{A}$ is defined as follows: $(\sigma(S))_{j}=s_{j+1}(j=0, \pm 1, \pm 2, \cdots)$.

Here, $\sigma$ defines a dynamical system on $\sum_{A}$. This discrete dynamical system is called a symbolic dynamical system.

The required properties of the symbolic dynamical system are given as follows:

Property $1 \sigma$ has a countable infinity number of periodic points. 


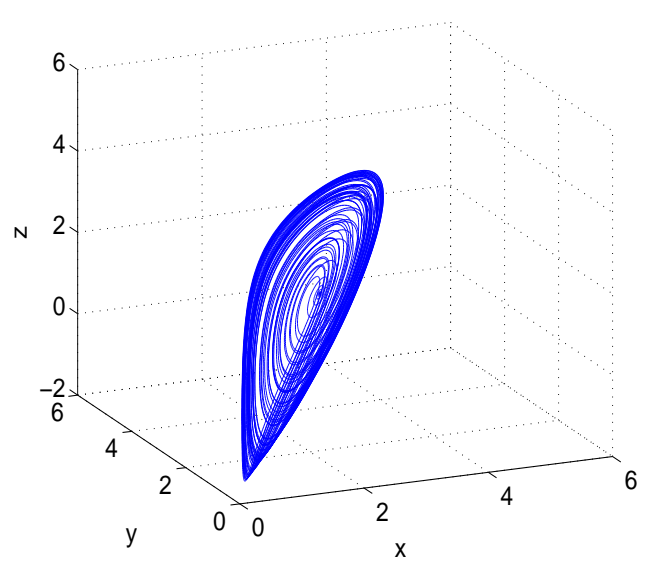

(a)

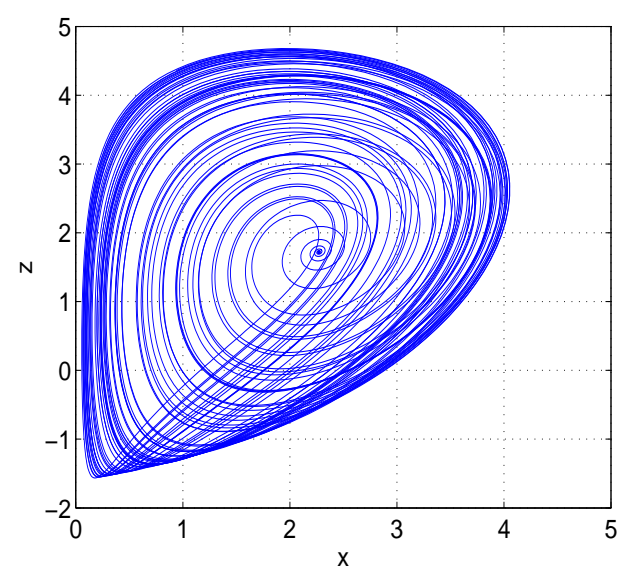

(c)

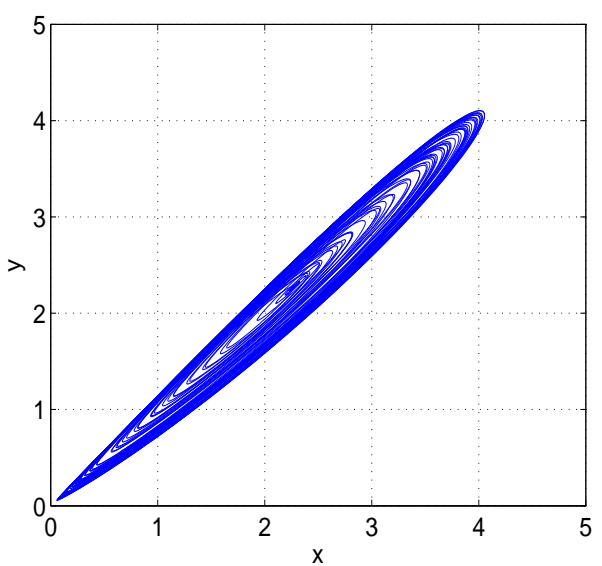

(b)

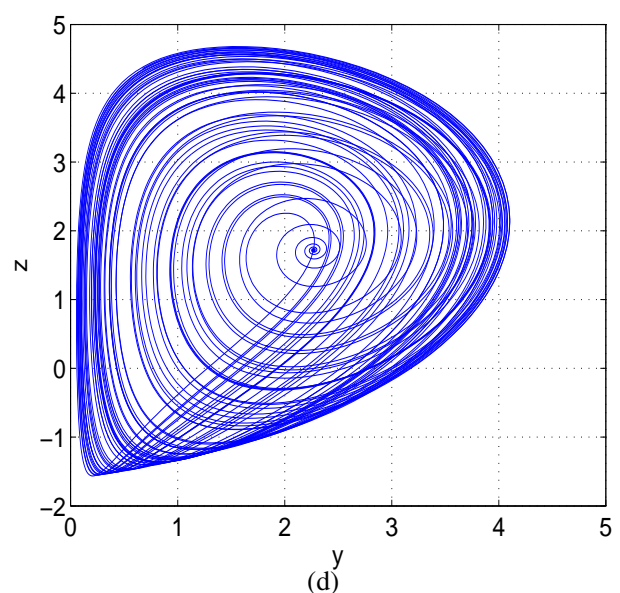

(d)

Fig. 1 Simulation of the single-scroll attractors about $O_{+}$in system (6) for $a=35, b=3, c=18.35978, k=2.85$, and $\tau=0.3$. (a) Three-dimensional phase space plot of the attractor; (b) the attractor projection on the $x-y$ plane; (c) the attractor projection on the $x-z$ plane; and (d) the attractor projection on the $y-z$ plane.

Property $2 \sigma$ has a dense set of periodic points in $\sum_{A}$.

Property $3 \sigma$ has a dense orbit, that is, there exists $\bar{S} \in \sum_{A}$ for which $\left\{S \mid S=\sigma^{k}(\bar{S}), k=0, \pm 1, \pm 2, \cdots\right\}$ is dense in $\Sigma_{A}$.

Property 4 There exists an uncountable set $\Delta \subset \sum_{A}-\operatorname{per}(\sigma)$, where per $(\sigma)$ represents all periodic point sets, so that

(1) $\lim _{n \rightarrow \infty} \sup d\left(\sigma^{n}(x), \sigma^{n}(y)\right) \geq 1, \forall x, y \in \Delta, x \neq y$;

(2) $\lim _{n \rightarrow \infty} \inf d\left(\sigma^{n}(x), \sigma^{n}(y)\right)=0, \forall x, y \in \Delta$; and

(3) $\lim _{n \rightarrow \infty} \sup d\left(\sigma^{n}(x), \sigma^{n}(y)\right) \geq 1, \forall x \in \Delta, \forall y \in \operatorname{per}(\sigma)$.

Property 5 The m-shift map $\sigma$ has topological entropy ent $(\sigma)=\log N$, where $N$ is the number of symbols in alphabet $A$. 


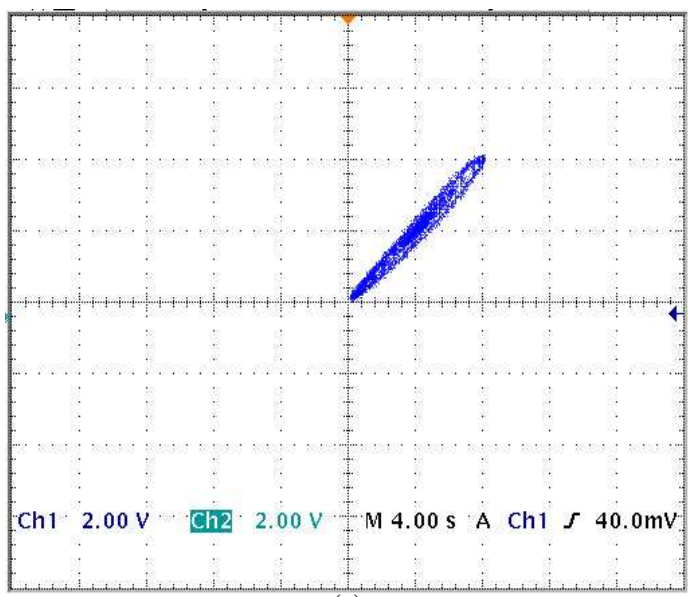

(a)

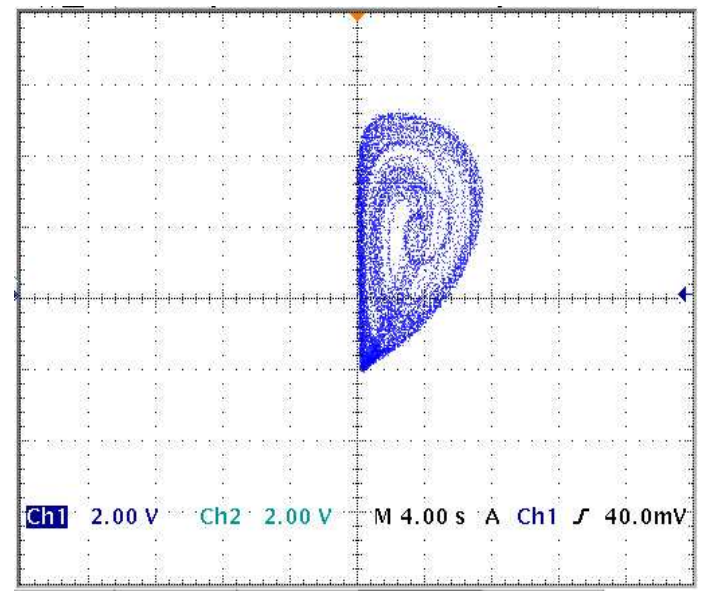

(b)

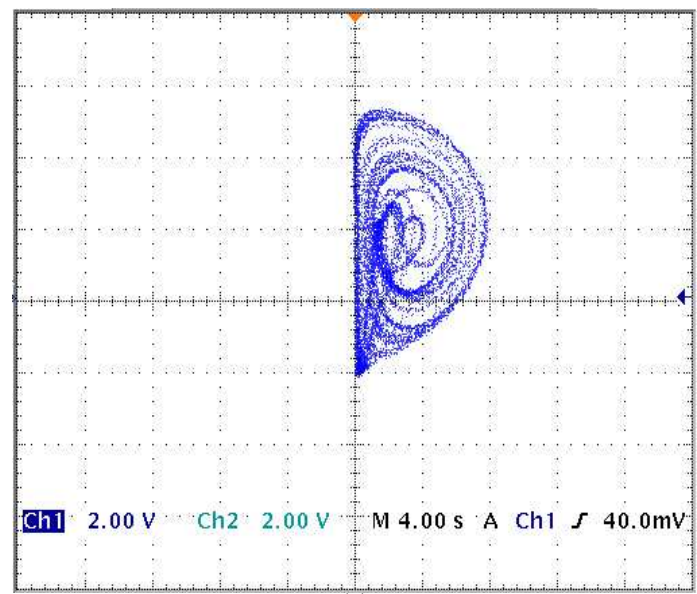

(c)

Fig. 2 The experimental result showing the single-scroll attractor of the Chen system with time delay. (a) The attractor projection on the $x-y$ plane, (b) the attractor projection on the $x-z$ plane, and (c) the attractor projection on the $y-z$ plane.

From the above properties, the $m$-shift map of $\sum_{A}$ has countable multiple dense periodic points and a dense orbit. It has sensitive dependent on the initial conditions in the Li-Yorke sense [31], and its topological entropy is larger than zero. All these properties are attributes of the chaotic system, and therefore, the symbolic dynamical system is a fundamental mathematical model of chaos.

\subsection{Topological Horseshoe Lemma}

The topological horseshoe theory is a strict mathematical theory to prove that chaos indeed exists in a system. The Topological Horseshoe Lemma provides conditions for the existence of a topological horseshoe. In this paper, we use this lemma to show the existence of a topological horseshoe in the Chen system with time delay. Some basic concepts are given as follows.

Let $X$ be a metric space; $D$, a compact subset in $X$; and $f: D \rightarrow X$, a map satisfying the assumption that there exist $m$ mutually disjoint compact subsets $D_{1}, \cdots, D_{m}$ of $D$, such that for each $D_{i}, f \mid D_{i}$ is continuous. 


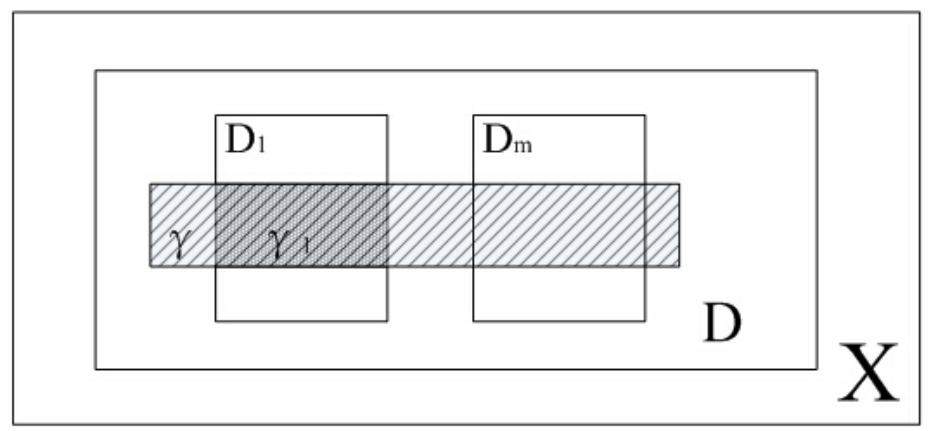

Fig. 3 Schematic diagram of the connection.

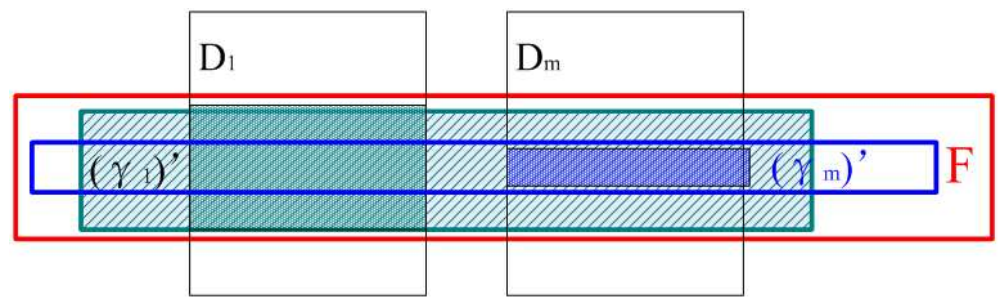

Fig. 4 Schematic diagram of a family of connections.

$f$-connected family: Let $\gamma$ be a compact connected subset of $D$ such that for each $1 \leq i \leq m, \gamma_{i}=\gamma \cap D_{i}$ is non-empty. Then, $\gamma$ is called a connection with respect to $D_{1}, \cdots, D_{m}$. If for $\gamma \in F, f\left(\gamma_{i}\right) \in F$, then $F$ is said to be an $f$-connected family with respect to $D_{1}, \cdots, D_{m}$.

Lemma 3.1 (Topological Horseshoe Lemma [9]) Suppose that there exists an $f$-connected family $F$ with respect to $D_{1}, \cdots, D_{m}$. Then, there exists a compact invariant set $K \subset D$, such that $F \mid K$ is semi-conjugate to $m$ - shift dynamics, therefore its entropy satisfies $h(f) \geq \log m$.

To apply the Topological Horseshoe Lemma in continuous dynamics, we need to construct a suitable Poincaré section $D, D \in X$ [7]. The Poincaré map is defined as follows: $P: D \rightarrow X$, where $D_{1}, \cdots, D_{m}$ are $m$ mutually disjoint compact subsets. Under the Poincaré map, each $D_{i}$ is continuous.

Figure 3 shows a schematic diagram of a connection, with the shaded rectangles $\gamma_{1}=\gamma \cap D_{1}$ corresponding to the connection of $D_{1}$, where $\gamma$ is a compact subset in $X$. Figure 4 shows a schematic diagram of a connected family. The $\left(\gamma_{1}\right)^{\prime}$ box filled with green diagonal stripes represents a connection corresponding to $D_{1}$; the $\left(\gamma_{m}\right)^{\prime}$ blue box represents a connection corresponding to $D_{m}$; and the $F$ red box represents a connection corresponding to $D_{1}, \cdots, D_{m}$ satisfying $\gamma \in F$ and $P\left(\gamma_{i}\right) \in F$ ( $P$ is the Poincaré map). If the relative position is similar to that shown in the diagram, then $F$ is called a $P$-connected family corresponding to $D_{1}, \cdots, D_{m}$.

Definition 1. For each $1 \leq i \leq m$, let $D_{i}^{1}$ and $D_{i}^{2}$ be two fixed disjoint compact subsets of $D_{i}$. A connected subset l of $D_{i}$ is said to connect $D_{i}^{1}$ and $D_{i}^{2}$ if $l \cap D_{i}^{1} \neq \emptyset$ and $l \cap D_{i}^{2} \neq \emptyset$, and we denote this by $D_{i}^{1} \stackrel{l}{\leftrightarrow} D_{i}^{2}$.

Definition 2. Let $l$ be a compact subsets of $D_{i}$. If l contains a connected subset $l^{\prime}$ such that $D_{i}^{1} \stackrel{f\left(l^{\prime}\right)}{\leftrightarrow} D_{i}^{2}$, we say that $f(l)$ across $D_{j}$ with $D_{j}^{1}$ and $D_{j}^{2}$, and this is denoted as $f(l) \mapsto D_{j}$. If $f(l) \mapsto D_{j}$ for every connected subset $l \subset D_{i}$ with $D_{i}^{1} \stackrel{l}{\leftrightarrow} D_{i}^{2}, f\left(D_{i}\right)$ is suitably across $D_{j}$ with respect to two pairs $D_{i}^{1}, D_{i}^{2}$ and $D_{j}^{1}$, $D_{j}^{2}$, and this is denoted as $f\left(D_{i}\right) \mapsto D_{j}$.

Lemma 3.2: If the map $f: D \rightarrow X$ satisfies the following assumptions:

(i) there exist $m$ mutually disjoint compact subsets $D_{1}, D_{2}, D_{3}, \ldots, D_{m}$ of $D$, and $f \mid D_{i}$ is continuous, and

(ii) the relation $f\left(D_{i}\right) \mapsto D_{j}$ holds for every pair with $i, j$, where $1 \leq i, j \leq m$,

then there exists a compact invariant set $K \subset D$ such that $f \mid K$ is semiconjugate to the full m-shift dynamics $\sigma \mid \sum_{m}$, and ent $(f) \geq \log m$. 
Corollary 1 If $f^{p}\left(D_{1}\right) \mapsto D_{1}$, then $f^{n p}\left(D_{1}\right) \mapsto D_{1}$, where $n$ is a positive integer.

Corollary 2 If $f^{p}\left(D_{1}\right) \mapsto D_{1}, f^{p}\left(D_{1}\right) \mapsto D_{2}$, and $f^{q}\left(D_{2}\right) \mapsto D_{1}$, then there exists a compact invariant set $K \subset D$ such that $f^{2 p+q} \mid K$ is semiconjugate to two-shift dynamics, and ent $(f) \geq(1 / 2 p+q) \log 2$.

The detailed proofs of these theorems and corollaries can be found in reference [8]. In the next section, we look for a connected family and prove the existence of chaos in the Chen system with time delay.

\section{Topological horseshoe of the Chen system with time delay}

In the Chen system with time delay, if a compact set $\Lambda$ satisfies the following the conditions:

(1) Points $x_{1}, x_{2}, \ldots, x_{k}$ on the orbit belongs to set $\Lambda$;

(2) There exists a Poincaré mapping crossing set $\Lambda$, and the Poincaré section have properties of topological horseshoe,we assert that $\Lambda$ is a chaotic attractor.

We first structure the corresponding Poincaré map using two different numerical methods, and then, we look for the topological horseshoe in the system.

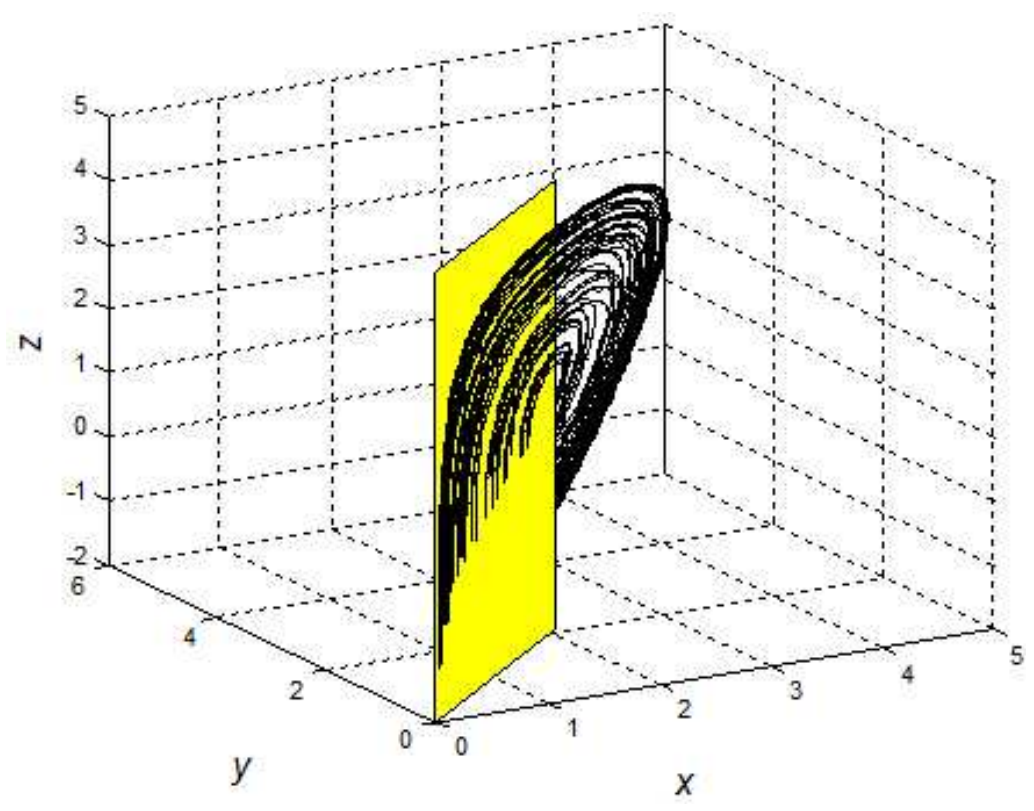

Fig. 5 The single-scroll attractors of the Chen experimental system with time delay and $A B C D$ plane.

\section{Method 1:}

We select a plane $A B C D$ with the normal vector $H=(1,-1,0)$ and the origin $(0,0,0)$ in it. In the twodimensional plane denoted as $A B C D$, coordinates are given by $(P x, P y)$ with origin at $(0,0,0)$, and the $y$ axis overlaps the $z$ axis in the original three-dimensional space. Select vertices in $A B C D$ given by $a=(0.838,1.16)$, $f=(0.874,1.18), m=(0.887,1.15), h=(0.851,1.13)$, as shown in Fig. 6(a).

Figure 6(a) shows the quadrilateral amfh. Let the Poincaré map be $P: a f m h \rightarrow A B C D$. When taking an arbitrary point $x \in$ afmh as the initial value, it is defined that $P(x)$ is the intersection point where the system trajectory crosses the plane $A B C D$ for the first time along the time forward direction.

It is shown that the four vertices of the quadrilateral afmh are mapped as $a^{\prime}, f^{\prime}, m^{\prime}, h^{\prime}$ by the Poincaré map, as shown in Fig. 6(b). Figure 7 shows schematically the relative position of the image after the Poincaré map and the original image, showing the map across. 


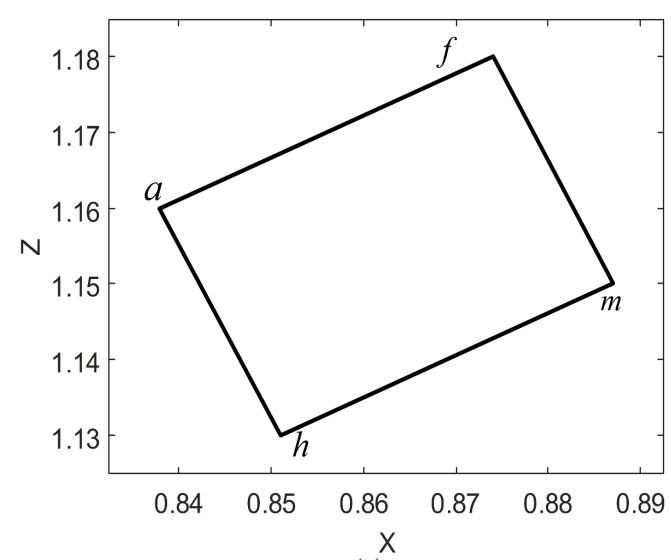

(a)

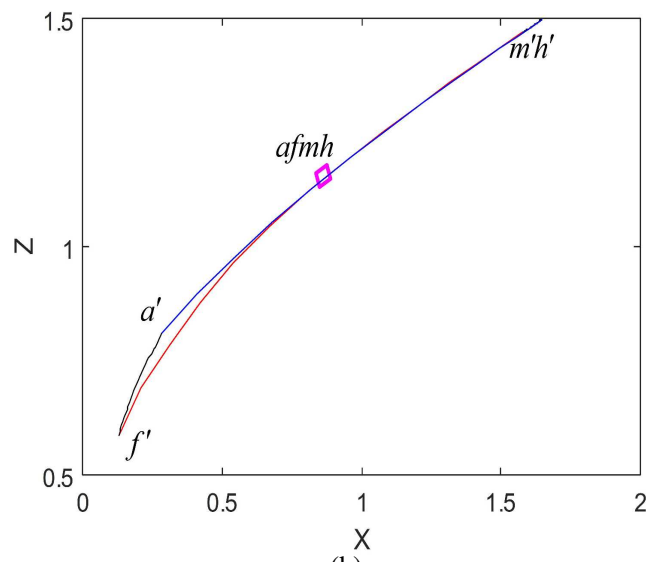

(b)

Fig. 6 The Poincaré section and its image under the Poincaré map. (a) the position of afmh points; (b) the position of afmh mapping points $a^{\prime} f^{\prime} m^{\prime} h^{\prime}$.

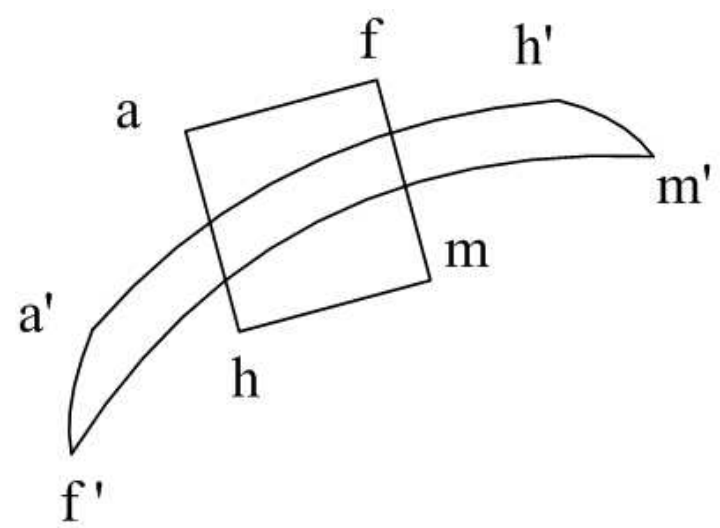

Fig. 7 Image $a^{\prime} f^{\prime} m^{\prime} h^{\prime}$ after the Poincaré map.

Then, we select two quadrilaterals $b c j i$ and delk on afmh. The coordinates of the four vertices of the quadrilateral $b c j i$ on the plane $A B C D$ are $b=(0.8432,1.163), c=(0.8508,1.167), j=(0.8639,1.137), i=$ $(0.8563,1.133)$.

The coordinates of the four vertices of the quadrilateral delk on plane $A B C D$ are $d=(0.8601,1.172)$, $e=(0.869,1.177), l=(0.8817,1.147), k=(0.873,1.142)$. Figure 8 shows the relative position of these two quadrilaterals intersecting the afmh one.

The simulation results show that two Poincaré maps of the two quadrilaterals $b c j i$ and delk are continuous. The image of the quadrilateral $b c j i$ under the Poincare map is the quadrilateral $b^{\prime} c^{\prime} j^{\prime} i^{\prime}$ and the image of the quadrilateral delk under the Poincaré map is the quadrilateral $d^{\prime} e^{\prime} l^{\prime} k^{\prime}$, as shown in Fig. 9.

Figure 10 shows the relative position between two quadrilaterals and their images under the Poincaré map, schematically showing the required mapping across.

As can be seen from the image of quadrilaterals $b c j i$ and delk under the Poincare map, the geometric properties satisfy the Topological Horseshoe Lemma. Therefore, a $P$-connected family exists. From the above criterion for the chaotic attractor, with the parameters $a=35, b=3, c=18.35978, k=2.85$, and $\tau=0.3$ and the corresponding Poincare map, there exists an invariant subset $K$ in the Chen system with time delay, and 


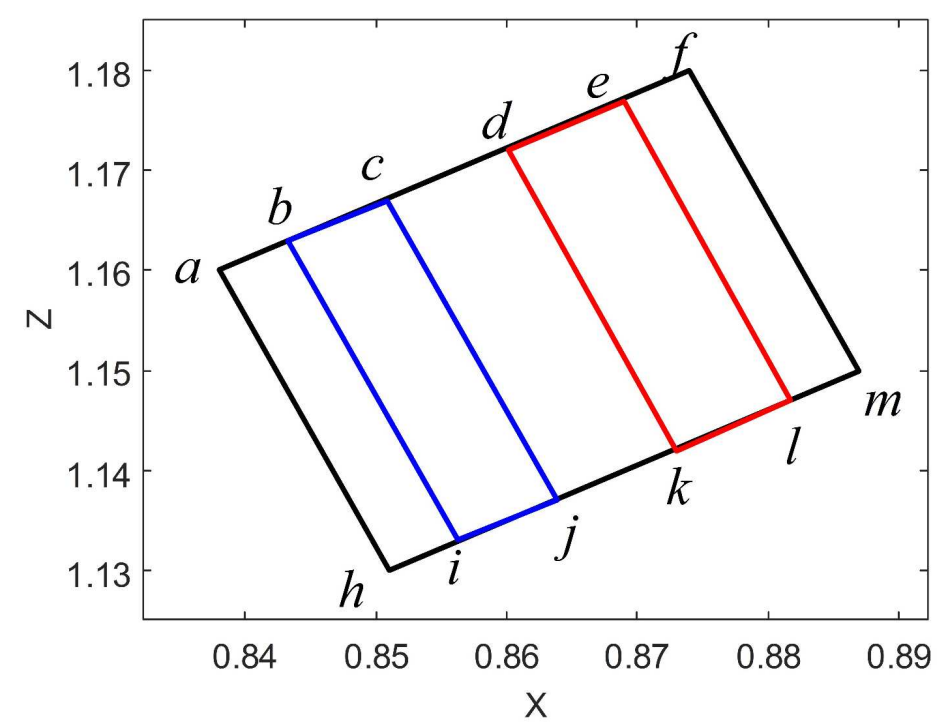

Fig. 8 The mutually disjoint closed subsets $b c j i$ and delk required by the topological horseshoe lemma.

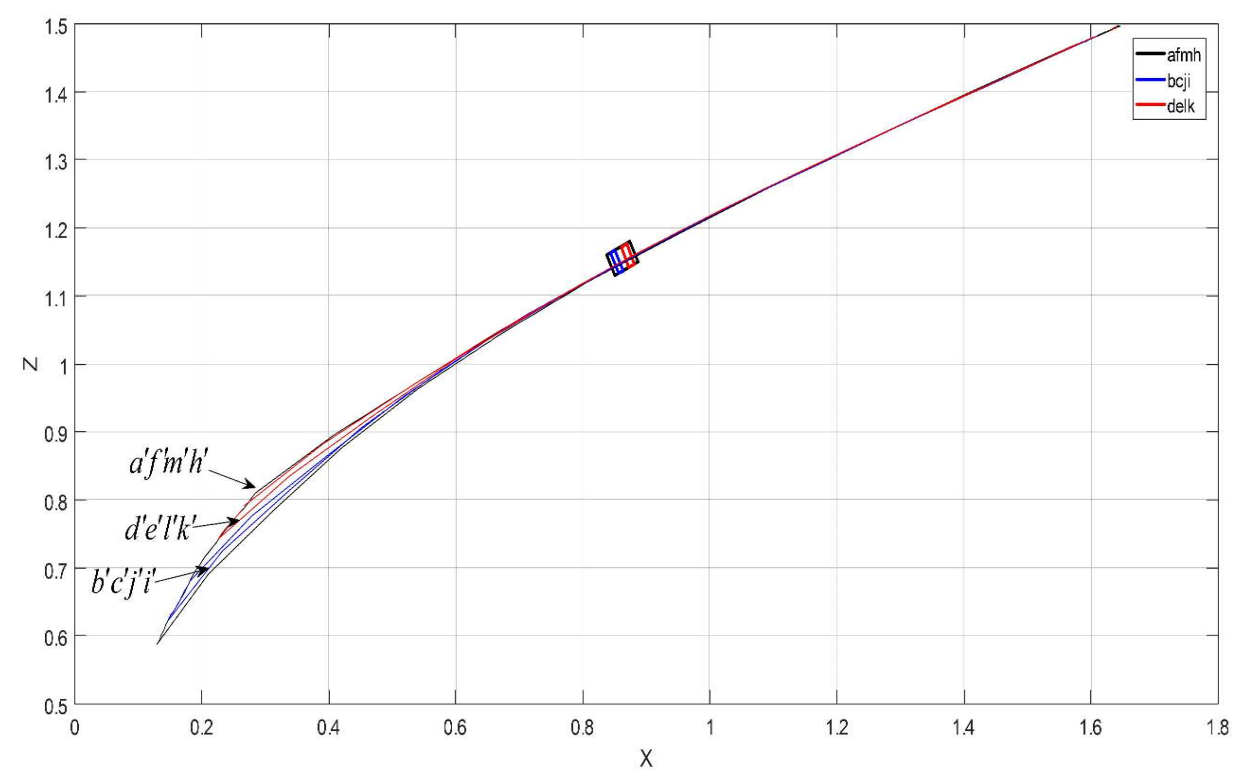

Fig. 9 The image of the quadrilateral bcji under the Poincaré map. (a) Global view of relative position of points and their mapping points; (b), (c), and (d) local enlarged images of areas PS4, PS5, and PS6, respectively.

the Poincare map, $P$, is restricted on the $K$ semi-conjugate with the two-shift map. We can conclude that the single-scroll attractor of the Chen system with time delay is a chaotic attractor.

Method 2: 


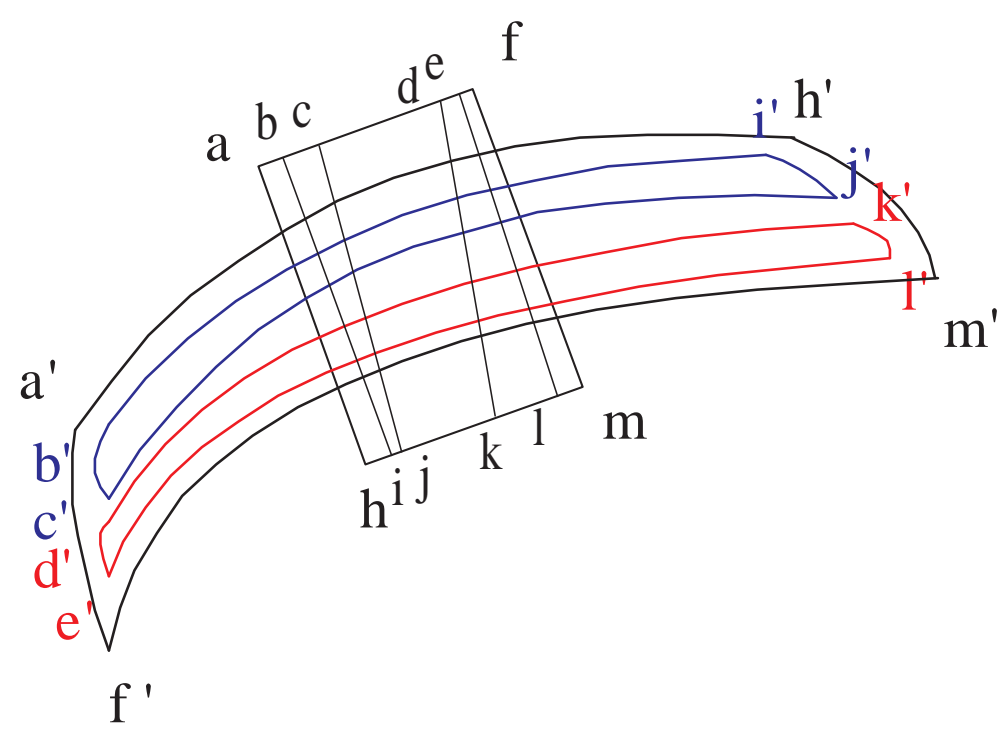

Fig. 10 Image of quadrilaterals $b c j i$ and delk under the Poincaré map.

Although the Method 1 finds topological horseshoes, it is time-consuming to identify the quadrilateral regions that maps the connections correctly. In the following, we use Corollaries 1 and 2 to find the horseshoe.

Step 1: Because the Poincaré section is already known, we can use the following method to find unstable periodic orbits (UPOs) on the Poincaré section.

Method for calculating UPOs: Let $\xi$ be the discrete sequence, $\xi_{k}$ be a point on $\xi, l$ be a positive integer, and $d_{k}=\left\|\xi_{k+l}-\xi_{k}\right\|$ be the Euclidean distance between $\xi_{k}$ and $\xi_{k+l}$. If $d_{k}$ is less than a sufficiently small constant $\varepsilon$, then there probably exists a short UPO of period $l$ near $\xi_{k}$.

We take $l=2$ and $\varepsilon=0.05$ as an example, and we obtain the location of four approximate UPOs of period 2, as shown by pair of red cross, pair of red pentagram, pair of black circle, pair of green pentagram in Fig. 11.

Step 2: Take a subset $D_{1}$ that is across itself under $P^{p 1}$.

First, let the quadrilateral $D_{1}$ be a small region, containing the UPOs. Then, adjust the vertices of $D_{1}$ until $D_{1}$ is suitably mapping across itself under $P^{p}$, where $p=2 n * l,(n=1,2,3 \ldots)$. If this is not possible, try another area containing short-period UPOs.

The vertex coordinates of the quadrilateral $D_{1}$ are selected as $(3.45,2.23),(3.5,2.26),(3.3,2.45),(3.25,2.42)$.

Select $p=16$. Denote the four edges of $D_{1}$ as $D_{1}^{1}, D_{1}^{2}, D_{1}^{3}$, and $D_{1}^{4}$.

The green solid-dot and black cross on the $D_{1}^{1}$ and $D_{1}^{2}$ under $p=16$ times Poincare map were indicated by the same marks on $P^{p}\left(D_{1}^{1}\right)$ and $P^{p}\left(D_{1}^{2}\right)$ in Fig. 12, and the blue line and red line indicates the map of $D_{1}^{3}$ and $D_{1}^{4}$.

\footnotetext{
${ }^{1}$ Here, $P^{p}$ represents the $p$-times Poincaré map.
} 


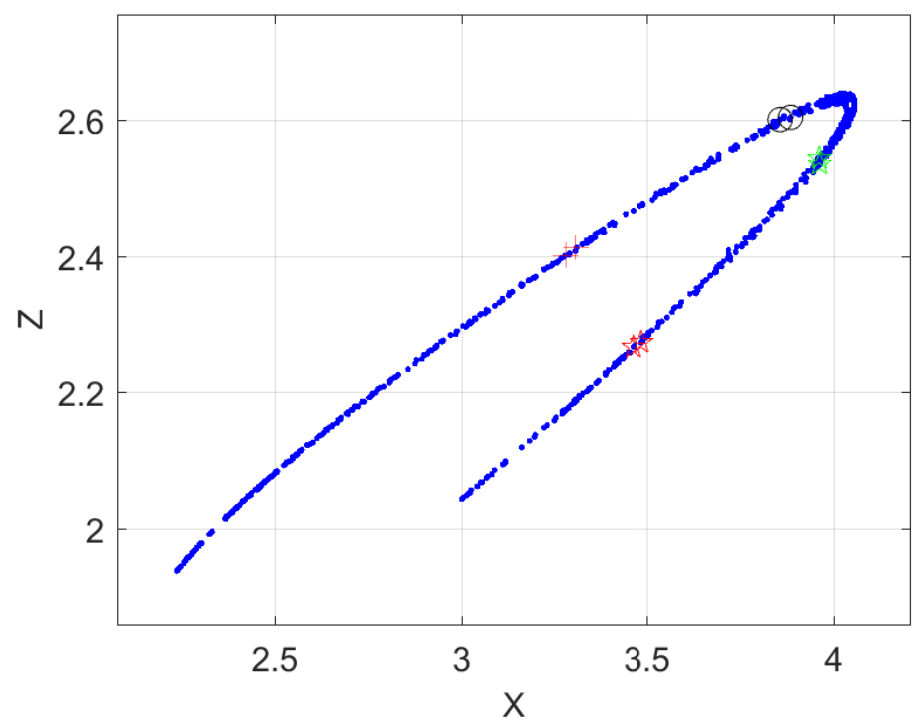

Fig. 11 Poincaré map and period 2 of the delayed Chen system.

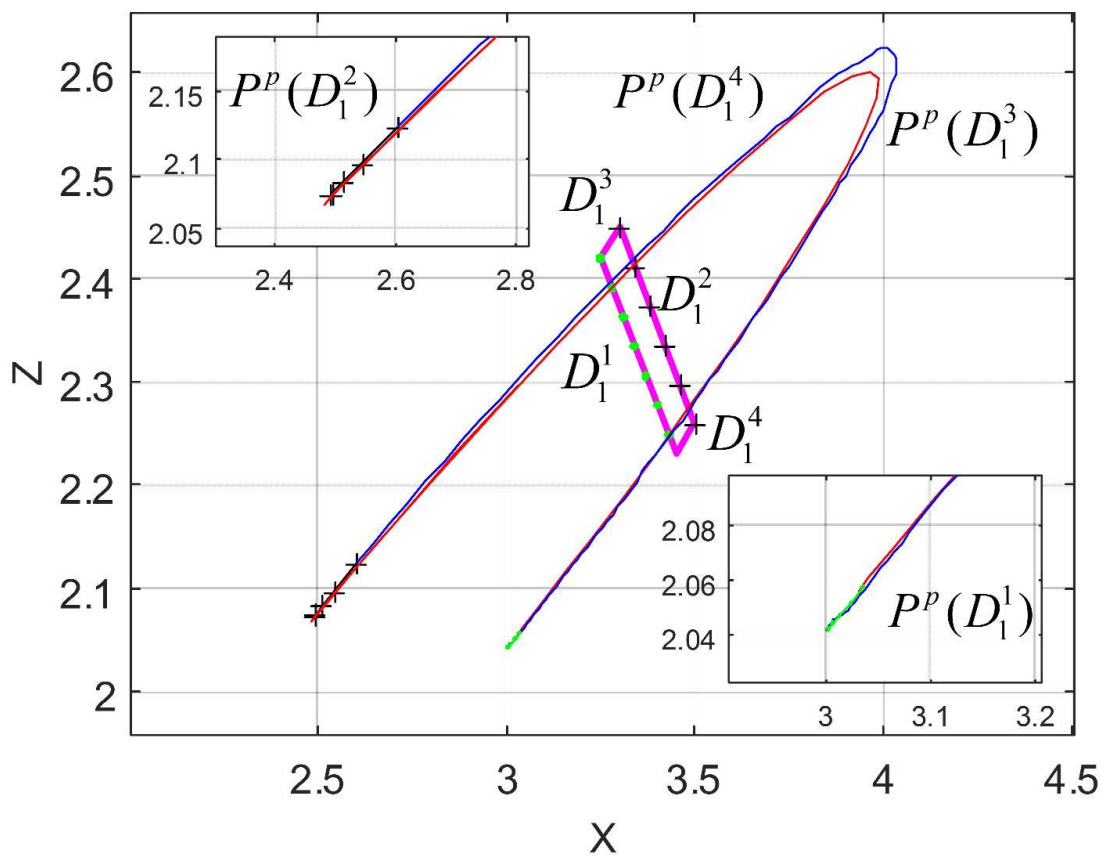

Fig. 12 The selection of $D_{1}$, and its map under $P^{p}$

Step 3: Choose a subset $D_{2}$ such that $P^{p}\left(D_{1}\right) \mapsto D_{2}$ and $P^{q}\left(D_{2}\right) \mapsto D_{1}$. 
For any $x_{k} \in X$, let $x_{k-q}=P^{-q}\left(x_{k}\right)$. If $S_{D_{1}}$ be the points on $D_{1}$, through inverse iteration, we obtain $P^{-q}\left(S_{D_{1}}\right)$. In particular, it usually contains several segments; select a segment among these, and denote a subset around it as $D_{2}$. If $D_{2}$ does not satisfy the condition $P^{-q}\left(D_{2}\right) \mapsto D_{1}$, try another segment. Here, $q=12$.

The vertex coordinates of the satisfactory quadrilateral $D_{1}$ are $(3.91,2.283),(3.9455,2.505),(3.911,2.63)$, $(3.87,2.62)$.

Denote the four edges of the $D_{2}$ as $D_{2}^{1}, D_{2}^{2}, D_{2}^{3}$, and $D_{2}^{4}$.

The green solid-dot and black cross in Fig. 13 indicate the map of $D_{2}^{1}$ and $D_{2}^{2}$ under map $P^{-q}$, and the blue line and red line indicate the image of $D_{2}^{3}$ and $D_{2}^{4}$ under $P^{-q}$.

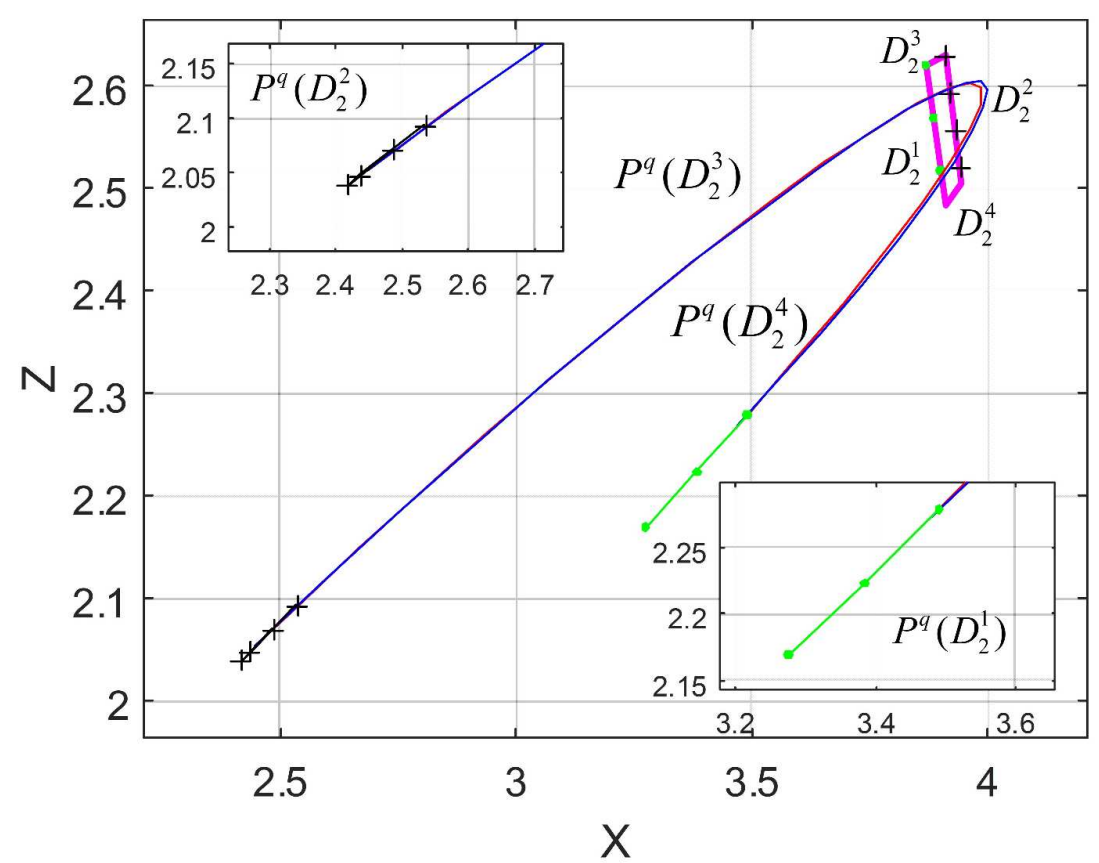

Fig. 13 Select $D_{2}$, and its map under $P^{-q}$.

As shown in Figs.12-13, the quadrilaterals $D_{1}$ and $D_{2}$ satisfy the corollary: $P^{16}\left(D_{1}\right) \mapsto D_{1}, P^{16}\left(D_{1}\right) \mapsto D_{2}$, and $P^{-12}\left(D_{2}\right) \mapsto D_{1}$. Consequently, the topological entropy of the system is not less than $\frac{1}{44} \log 2$.

The key step of method 2 is the selection of $D_{2}$. Based on the known condition $P^{p}\left(D_{1}\right)$, it is easy to find $D_{2}$ such that $P^{p}\left(D_{1}\right) \mapsto D_{2}$; however, this does not mean that $P^{q}\left(D_{2}\right) \mapsto D_{1}$. Method 2 for inverse mapping has few segments at most; therefore, we can perform a finite number of trials until we obtain the topological horseshoe. This method thus avoids the large number of trials required when using Method 1. Consequently, Method 2 is simpler and easier to operate without homological techniques.

\section{Conclusions}

By using the Topological Horseshoe Lemma and numerical simulations, we prove the existence of a topological horseshoe in the Chen system with time delay, and, therefore, we prove that the single-scroll attractor is indeed chaotic. We also demonstrate that the topological entropy of the single-scroll attractor is larger than $\frac{1}{44} \log 2$. 
Acknowledgements This work was supported in part by the NSFC (61172070 and 60804040), Innovative Research Team of Shaanxi Province (2013KCT-04), and Fok Ying Tong Education Foundation Young Teacher Foundation (111065). Key program of Natural Science Foundation of Shaaxi Province (2016ZDJC01)

\section{References}

1. N. Levinson, A Second Order Differential Equation with Singular Solutions, Annals Of Mathematics, 50, 127-153 (1949)

2. Steven Smale, Differentiable dynamical systems, Bull. Amer. Math. Soc, 73, 747-817 (1967)

3. Celso Grebogi, Edward Ott, James A. Yorke, Crises, sudden changes in chaotic attractors, and transient chaos, Physica D Nonlinear Phenomena, 7, 181-200 (1983)

4. Colin Sparrow, The Lorenz Equations: Bifurcations, Chaos and Strange Attractors. Applied Mathematical Sciences, 41, Springer-Verlag, (1982)

5. M.Rychlik, Lorenz attractors through a ShilnikovCtype bifurcation, Ergod Theory and Dyn. Sys., 10, 811-C840, (1989)

6. Warwick Tucker, The Lorenz attractor exists, C. R. Acad. Sci. Paris, 328, Série I, 1197-1202 (1999)

7. Kennedy Judy, York James, Topological horseshoes, Tran. Amer. Math. Soc., 353, 2513-2530 (2001)

8. Xiao Song Yang, Yun Tang, Horseshoes in piecewise continuous mappings, Chaos, Solitons \& Fractals, 19, 841-845 (2004)

9. Xiao Song Yang, Topological horseshoes and computer assisted verification of chaotic dynamics, International Journal of Bifurcation and Chaos, 19, 1127-1145 (2009)

10. Xiao Song Yang, Qing Du Li, A computer-assisted proof of chaos in Josephson junctions, Chaos, Solitons \& Fractals, 27, 25-30 (2006)

11. Chun Lai Li, Lei Wu, Hong Min Li, Yao Nan Tong, A novel chaotic system and its topological horseshoe, Nonlinear Analysis: Modelling and Control , 18, 66-77 (2013)

12. Yan Huang, Xiao Song Yang, Horseshoe in modified Chen's attractors, Chaos, Solitons \& Fractals, 26, 79-85 (2005)

13. Kui Biao Deng, Si Min Yu, Estimating ultimate bound and finding topological horseshoe for a new chaotic system, Optik International Journal for Light and Electron Optics, 125, 6044-6048 (2014)

14. Wen Juan Wu, Zeng Qiang Chen, Zhu Zhi Yuan, A computer-assisted proof for the existence of horseshoe in a novel chaotic system, Chaos, Solitons \& Fractals, 41, 2756-2761 (2009)

15. Qing Du Li, Hong Zheng Zeng, Xiao Song Yang, On hidden twin attractors and bifurcation in the Chua's circuit, Nonlinear Dynamics, 77, 255-266 (2014)

16. Jianbin He, Simin Yu, Jianping Cai, Topological horseshoe analysis for a three-dimensional anti-control system and its application, Optik, 127, 9444-9456 (2016)

17. Leilei Zhou, Zengqiang Chen, Zhonglin Wang, Jiezhi Wang, On the analysis of local bifurcation and topological horseshoe of a new 4D hyper-chaotic system, Chaos, Solitons \& Fractals, 91, 148-156 (2016)

18. Lei Wang, Xiao Song Yang, Topological horseshoe and its uniform hyperbolicity in the HP model, Journal of Mathematical Biology, 75, 129-143 (2017)

19. Zambrano-Serrano E, Campos-Cantón E, Munoz-Pacheco J M, Strange attractors generated by a fractional order switching system and its topological horseshoe, Nonlinear Dynamics , 83, 1629-1641 (2016)

20. Zhonglin Wang, Leilei Zhou, Zengqiang Chen, Jiezhi Wang, Local bifurcation analysis and topological horseshoe of a 4D hyper-chaotic system, Nonlinear Dynamics, 83, 2055-2066 (2016)

21. Qing Du Li, A topological horseshoe in the hyperchaotic Rössler attractor, Physics Letters A, 372, 2989-2994 (2008)

22. Qing Du Li, Song Tang, Algorithm for finding horseshoes in three-dimensional hyperchaotic maps and its application, Acta Physica Sinica (in Chinese), 62, 210510 (2013)

23. Hai Peng Ren, Chao Bai, Zhan Zhan Huang, Celso Grebogi, Secure Communication Based on Hyperchaotic Chen System with Time-Delay, International Journal of Bifurcation and Chaos, 27, 1750076 (2017)

24. Ya Qiong Jia, Guo Ping Jiang, Chaotic system synchronization of state-observer-based fractional-order time-delay, Acta Physica Sinica (in Chinese), 66, 160501 (2017)

25. G. Velmurugan, R. Rakkiyappan, Hybrid projective synchronization of fractional-order chaotic complex nonlinear systems with time delays, Journal of Computational and Nonlinear Dynamics, 11, 031016 (2016)

26. Hai Peng Ren, Chao Bai, Kun Tian, Celso Grebogi, Dynamics of delay induced composite multi-scroll attractor and its application in encryption, International Journal of Non-Linear Mechanics, 94, 334-342 (2017)

27. Guan Rong Chen, Tetsushi Ueta, Yet another chaotic attractor, International Journal of Bifurcation and Chaos, 9, 1465-1466 (1999)

28. Hai Peng Ren, Ding Liu, Chong Zhao Han, Anticontrol of chaos via direct time delay feedback, Acta Physica Sinica (in Chinese), 55, 2694-2701 (2006)

29. Hai Peng Ren, Wen Chao Li, Heteroclinic orbits in Chen circuit with time delay, Communications in Nonlinear Science and Numerical Simulation, 15, 3058-3066 (2010)

30. Zeng Rong Liu, Perturbation Criteria for Chaos. Shanghai Scientific and Technological Education Publishing House(in Chinese), Shanghai (1994)

31. Tien Yien Li , James A. Yorke, Period three implies chaos, The American Mathematical Monthly, 82, 985-992 (1975) 